\title{
LIMITAÇÕES NUTRICIONAIS PARA A CULTURA DO ARROZ IRRIGADO EM SOLO ORGÂNICO DA REGIÃO NORTE FLUMINENSE ${ }^{(1)}$
}

\author{
W.E. de B. ANDRADE ${ }^{(2)}$, A.F. de SOUZA ${ }^{(3)}$ \& J .G. de CARVAL HO ${ }^{(4)}$
}

\begin{abstract}
RESUMO
As limitações nutricionais de um solo orgânico para a cultura do arroz irrigado por inundação, cv. Inca, foram identificadas em casa de vegetação em Lavras (MG) de dezembro/94 a junho/95. Coletou-se o material do solo utilizado na região Norte Fluminense, constituindo os tratamentos de testemunha (solo natural), completo (N, P, K, calcário, S, B, Co, Cu, Mo e Zn), e completo menos um nutriente de cada vez. Os resultados permitem concluir que a omissão de $\mathbf{N}$ e $\mathrm{K}$ reduziu a produção de matéria seca pela parte aérea do arroz, quando colhida na maturação dos grãos, em 28 e 24\%, respectivamente, em relação ao completo. Não se observou decrésci mo na matéria seca (maturação), quando houve omissão de fósforo, calcário, enxofre e micronutrientes da adubação. Na ausência de N, K eZn, o acúmulo desses nutrientes na parte aérea das plantas de arroz foi inferior ao do tratamento completo.
\end{abstract}

Termos de indexação: limitações nutricionais, várzea, solo orgânico, Oryza sativa.

\section{SUMMARY: NUTRITIONAL LIMITATIONS OF RICE CROP IN AN ORGANIC SOIL OF NORTHERN FLUMINENSE REGION}

The nutritional limitations of an organic soil supporting a lowland rice crop, cv. Inca, were identified in a greenhouse experiment in Lavras, State of Minas Gerais, from December, 1994, to J une, 1995. Soil samples were collected in Northern Fluminense region. Treatments consisted of control (natural soil), complete (N, P, K, limestone, S, B, Co, Cu, Mo, and Zn) and compl etelacking one of these nutrients at a time At the grain maturation time the treatments lacking $\mathrm{N}$ and $\mathrm{K}$ showed a shoot dry matter decrease of 28 and $24 \%$, respectively, compared to the complete treatment. No decrease was observed in the absence of $\mathrm{P}, \mathrm{S}$, limestone and micronutrients. Theabsence of $\mathrm{N}, \mathrm{K}$ and $\mathrm{Zn}$ resulted in a lower accumulation of thesenutrients in shoot dry matter when compared to the complete treatment.

Index terms: nutritional constraint, Iowland, organic soil, Oryza sativa.

\footnotetext{
(1) Recebido para publicação em dezembro de 1995 e aprovado em maio de 1997.

(2) Engenheiro-Agrônomo, Pesquisador da PESAGRO-RI O/E EC. Avenida Francisco Lamego, 134. Bairro Guarus. CEP $28080-000$ Campos (RJ).

(3) Professor Titular do Departamento de Agricultura da Universidade Federal de Lavras (DAG/UFLA). Caixa Postal 37, CEP 37200000 Lavras (MG).

(4) Professor Titular do Departamento de Ciência do Solo (DCS/UFLA). Caixa Postal 37, CEP 37200-000 Lavras (MG). Bolsista do CNPq.
} 


\section{INTRODUÇÃO}

Apesar da importância das áreas de várzea, existem poucas informações em termos de capaci dade desses solos em suprir nutrientes para as culturas e permitir alta produtividade (Abreu \& Lopes, 1987). Experimentos com a cultura do arroz irrigado em tais solos de São Paulo e Minas Gerais mostraram que nitrogênio, fósforo e potássio têm limitado a produção freqüentemente (Blanco et al., 1964; Leite et al., 1970a,b; Novais et al., 1972; Pereira, 1985; Guilherme, 1990; Paula et al., 1990; Bastos, 1993; Mesquita, 1993).

As respostas a $\mathrm{N}$ ocorreram mesmo com o teor médio de matéria orgânica do solo acima do considerado al to (Comissão de Fertilidade do Solo do Estado de Minas Gerais, 1989). Abreu \& Lopes (1987) e Paula et al. (1990), em sol os gl ei húmico e orgânico de Minas Gerais, concluíram que o teor de matéria orgânica é um parâmetro inadequado de previsão de resposta das culturas de milho e arroz à adubação nitrogenada. As respostas a fósforo e a potássio também ocorreram, apesar de os teores iniciais no solo estarem acima dos valores considerados altos pela citada Comissão. J á para os micronutrientes, os dados experimentais obtidos em sol os de várzeas são poucos, o que não permite uma análise mais apurada de seus efeitos na nutrição mineral do arroz. Entretanto, segundo Paula et al. (1990), a omissão de Zn e Cu contribuiu para reduzir a produção de arroz em solo glei húmico. Com relação à calagem, os estudos envolvendo solos cultivados com arroz irrigado por inundação não são concl usivos, em função do aumento do $\mathrm{pH}$ pelas reações de redução que ocorrem, sendo relatados tantoresultados positivos (Guilherme, 1990) quanto negativos (Pereira, 1985).

Por esses resultados, verifica-se a variabilidade das características químicas que interferem na obtenção de maior produtividade nas várzeas, sugerindo, para cada tipo de solo, estudos específicos que viabilizem sua utilização. $O$ presente experimento teve por objetivo avaliar a fertilidade de um solo orgânico, empregando a técnica do el emento faltante.

\section{MATERIAL E MÉTODOS}

O experimento foi instalado em casa de vegetação do Departamento de Agricultura da Universidade Federal de Lavras (DAG/UFLA), em vasos com $5 \mathrm{~kg}$ desolo, por meio da técnica da diagnose por subtração (Sanchez, 1976).

Foi utilizado um solo orgânico(5) de Macaé (RJ ), sendo as amostras coletadas na profundidade de $0-20 \mathrm{~cm}$. As características determinadas foram as seguintes: $\mathrm{pH}(1: 2,5)$; $\mathrm{P}\left(43 \mathrm{mg} \mathrm{dm}^{-3}\right)$; $\mathrm{K}$ (117 $\left.\mathrm{mg} \mathrm{dm}^{-3}\right)$; Ca $\left(27 \mathrm{mmol}_{\mathrm{c}} \mathrm{dm}^{-3}\right) ; \mathrm{Mg}\left(16 \mathrm{mmol}_{\mathrm{c}} \mathrm{dm}^{-3}\right) ; \mathrm{Al}$ $\left(27 \mathrm{mmol}_{\mathrm{c}} \mathrm{dm}^{-3}\right) ; \mathrm{Na}\left(1,0 \mathrm{mmol}_{\mathrm{c}} \mathrm{dm}^{-3}\right) ; \mathrm{H}+\mathrm{Al}$ (186 mmol $\left.\mathrm{cm}^{-3}\right)$; $\mathrm{MO}\left(213,0 \mathrm{~g} \mathrm{~kg}^{-1}\right) ; \mathrm{S}\left(154,54 \mathrm{mg} \mathrm{dm}^{-3}\right)$;

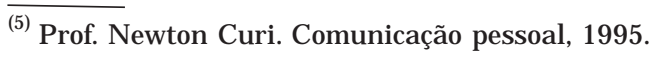

B (0,20 mg dm-3); Cu (1,59 mg dm-3); Fe (357,39 mg dm-3); Zn (4,34 mg dm-3); Mn (26,93 mg dm-3); argila (480 $\left.\mathrm{g} \mathrm{kg}^{-1}\right)$; areia (80 $\left.\mathrm{g} \mathrm{kg}^{-1}\right)$; silte $\left(440 \mathrm{~g} \mathrm{~kg}^{-1}\right)$; $\mathrm{dp}\left(1,78 \mathrm{~g} \mathrm{~cm}^{-3}\right)$ eds $\left(0,56 \mathrm{~g} \mathrm{~cm}^{-3}\right)$. Na caracterização química, foram determinados $\mathrm{pH}$ em água, $\mathrm{P}, \mathrm{K}, \mathrm{Ca}, \mathrm{MgeAl}$, conforme descrito em Vettori (1969), com modificações da EMBRAPA (1979), e o H + Al segundo Raij et al. (1987). O B foi estabelecido pelo método da água quente e no extrato, de acordo com o método de curcumina (J ackson, 1970). Fe, Mn, Cu e Zn foram determinados no extrato de Mehlich 1 por espectrofotometria de absorção atômica; a matéria orgânica, segundo Raij et al. (1987), e os teores de $\mathrm{SO}_{4}{ }^{2-}$, segundo Blanchar et al. (1965). O solo col etado foi seco ao ar e passado em peneira com malha de cinco milímetros para homogeneização.

Os tratamentos consistiram em testemunha (solo natural ), completo ( $\mathrm{N}, \mathrm{P}, \mathrm{K}$, calagem, S, B, Co, Cu, Mo e $\mathrm{Zn}$ ) e completo menos um nutriente de cada vez, totalizando doze tratamentos. O delineamento foi de blocos casualizados, com quatro repetições. Para a adubação de plantio, seguiu-se recomendação de Malavolta \& Muraoka (1985) e El-Husny (1992), com al gumas adaptações (Quadro 1). O fósforo e o cal cário foram previamente incorporados ao solo (30 dias), sendo os demais nutrientes aplicados via solução nutritiva, após o desbaste. Na calagem, utilizou-se cal cário cal cinado ( $40 \%$ de $\mathrm{CaO}, 15 \%$ de M gO ePRNT de $92 \%$ ), com dose determinada pelo método de neutralização do alumínio. A umidade do solo foi mantida a $80 \%$ do vol ume total de poros, empregandose água desmineralizada, durante 15 dias após o desbaste; a partir daí, o sol o foi saturado, mantendose uma lâmina d'água de $4 \pm 0,5 \mathrm{~cm}$. A planta teste foi o arroz, cv. Inca, sendo semeadas, em 27/12/94, dez sementes por vaso. A pós o desbaste, foram mantidas três plantas por vaso, sendo uma col hida na floração e as demais, levadas até à maturação dos grãos. A adubação de cobertura foi feita na diferenciação do primórdio floral. $\mathrm{Na}$ col heita, as plantas foram cortadas rente ao sol o e a parte aérea levada a secar em estufa a $60^{\circ} \mathrm{C}$ até peso constante.

\section{Quadro 1. Doses e fontes de nutrientes aplicados em solo orgânico contido em vasos com capacidade para 5 kg}

\begin{tabular}{ccl}
\hline Nutriente & Adubação de plantio & \multicolumn{1}{c}{ Fonte } \\
\hline \multirow{3}{*}{$\mathrm{mg} \mathrm{kg}^{-1}$ de solo } & \\
$\mathrm{N}$ & 200 & Uréia \\
$\mathrm{P}$ & 111 & Ácido fosfórico \\
$\mathrm{K}$ & 200 & Cloreto de potássio \\
$\mathrm{S}$ & 36 & Sulfato de potássio \\
& - & Sulfato de amônio \\
$\mathrm{B}$ & 0,5 & Ácido bórico \\
$\mathrm{Cu}$ & 1,5 & Cloreto cuproso \\
Mo & 0,1 & Ádido molíbdico \\
$\mathrm{Zn}$ & 2,5 & Cloreto de zinco \\
Co & $7,6.10^{-5}$ & Cloreto de cobalto \\
& Adubação de cobertura & \\
$\mathrm{N}$ & 50 & Uréia \\
$\mathrm{K}$ & 50 & Cloreto de potássio \\
\hline
\end{tabular}


Foram feitas análises para as características obtidas na maturação do grão, a saber: número de perfilhos e de panículas/planta, altura de planta, matéria seca da parte aérea e quantidade acumulada de $\mathrm{N}, \mathrm{P}, \mathrm{K}, \mathrm{Ca}, \mathrm{Mg}, \mathrm{S}, \mathrm{B}, \mathrm{Zn}, \mathrm{Fe}, \mathrm{Mn}$ e Cu na parte aérea. As médias dos tratamentos foram comparadas, utilizando-se o teste deTukey ao nível de 5\%, conforme Pimentel Gomes (1984). As concentrações de Ca, Mg, $\mathrm{Cu}, \mathrm{Fe}, \mathrm{Mn}$ e Zn nos extratos foram determinadas por espectrofotometria de absorção atômica; a de K, por fotometria de chama; a de P, por colorimetria usando vanadato-molibdato; a de S, por turbidimetria; a de $\mathrm{N}$, pelo método microK jeldahl, sendo o $\mathrm{B}$ extraído por via seca (incineração).

\section{RESULTADOS E DISCUSSÃO}

Pretendia-se conduzir o arroz até à maturação do grão, quando poderia ser quantificado o efeito dos diferentes tratamentos na produção final. E ntretanto, houve grande chochamento de grãos, atribuído à elevada temperatura na casa de vegetação, superior a $42^{\circ} \mathrm{C}$ em três dias seguidos e por período superior a duas horas, observada na diferenciação dos primórdios florais. Segundo Andrade (1991), temperaturas superiores a 38C são consideradas prejudiciais nessa fase. Como não foi possível a colheita dos grãos, na fase final, determinou-se a matéria seca total da parte aérea. Segundo Paula et al. (1990), mediante os resultados de produção de matéria seca da parteaérea das plantas de arroz, é possível ter uma indicação dos elementos deficientes e a importância relativa dessa deficiência.

Pelo quadro 2, verifica-se que os maiores decréscimos de produção de matéria seca da parte aérea ocorreram na testemunha (38\%), seguida da omissão do N (28\%) e do K (24\%). Os demais tratamentos não diferiram, signicativamente, do completo. Com a omissão do N, a redução foi devida, em parte, ao menor número de perfilhos/planta e, na omissão do K, à menor altura de plantas. Não se observaram diferenças significativas entre esses tratamentos e o completo, em relação ao número de panículas/planta. Os resultados concordam, em parte, com os obtidos por outros autores (Blanco et al ., 1964; Leite et al., 1970ab; Novais et al., 1972; Paula et al., 1990), quanto à importância do nitrogênio e do potássio na produção do arroz em várzea. Blanco et al. (1964), avaliando a fertilidade de alguns solos de várzea do Vale do Paraíba para a cultura do arroz, encontraram respostas na produção de grãos ao N, seguido do P e do K. Resultados semelhantes foram obtidos por Paula et al. (1990) em solo glei húmico de Minas Gerais, em que a omissão de N, P eK reduziu a produção de matéria seca pelo arroz. Por outro lado, estudando solos de várzea em dez locais da Zona da Mata de Minas Gerais, N ovais et al . (1972) verificaram ser o fósforo o maior limitante da produção de grãos. Leite et al. (1970ab) observaram, em vários locais do Vale do Paraíba, aumentos significativos de produção de grãos devidos, principalmente, a N.

Para os micronutrientes, os resultados concordam com os encontrados por Leite et al. (1970b), que também não observaram respostas da cultura do arroz irrigado em solos de várzea paulista. Entretanto, Paula et al. (1990) observaram que a ausência de Zn e Cu contribuiu para reduzir a matéria seca do arroz em solo glei húmico de Lambari (MG).

No quadro 3 encontra-se a quantidade de nutrientes acumulada na parte aérea da planta. Na ausência de $\mathrm{N}$, foi significativamente menor sua acumulação, quando comparada à do tratamento completo, o que também justifica a redução na

Quadro 2. Número de perfilhos e de panículas por planta, altura de planta e acúmulo de matéria seca na parte aérea do arroz, colhido na maturação dos grãos

\begin{tabular}{|c|c|c|c|c|c|}
\hline Tratamentos & $\begin{array}{l}\text { Número de } \\
\text { perfilhos }\end{array}$ & $\begin{array}{l}\text { Número de } \\
\text { panículas }\end{array}$ & $\begin{array}{l}\text { Altura da planta } \\
\text { (cm) }\end{array}$ & $\begin{array}{l}\text { Matéria seca } \\
\text { (g) }\end{array}$ & $\begin{array}{c}\text { Matéria seca relativa } \\
(\%)\end{array}$ \\
\hline Completo & $26,88 a$ & $24,50 a b$ & $85,80 a b$ & $172,01 a$ & 100 \\
\hline Testemunha & $22,88 a b$ & $21,13 a b$ & $76,40 c$ & $106,59 d$ & 62 \\
\hline$-\mathrm{N}$ & $18,50 \mathrm{~b}$ & $17,63 b$ & $82,00 a b c$ & $123,43 \mathrm{~cd}$ & 72 \\
\hline$-P$ & $25,13 a b$ & $23,75 a b$ & $88,60 a$ & $173,35 a$ & 101 \\
\hline$-K$ & $27,75 a$ & $26,00 a$ & $80,50 b c$ & $131,16 \mathrm{bcd}$ & 76 \\
\hline$-S$ & $23,38 a b$ & $22,38 a b$ & $87,00 a b$ & $157,83 a b c$ & 92 \\
\hline -Calcário & $28,88 a$ & $27,75 a$ & $83,45 a b c$ & $153,58 a b c$ & 89 \\
\hline$-B$ & $24,88 a b$ & $22,75 a b$ & $86,45 a b$ & $155,30 a b c$ & 90 \\
\hline$-\mathrm{Cu}$ & $26,25 a$ & $24,13 a b$ & $88,28 a$ & $167,89 a b$ & 98 \\
\hline$-Z n$ & $28,13 a$ & $26,13 a$ & $88,70 a$ & $177,51 a$ & 103 \\
\hline- Mo & $27,38 a$ & $25,60 a$ & $88,20 a$ & $177,42 a$ & 103 \\
\hline$-\mathrm{Co}$ & $27,25 a$ & $26,00 a$ & $86,23 a b$ & $176,25 a$ & 102 \\
\hline
\end{tabular}

(1) Considerando-se o acúmulo de matéria seca no tratamento completo igual a 100.

Em cada coluna, as médias seguidas pela mesma letra não diferem, significativamente, pelo teste de Tukey ao nível de 5\%. 
Quadro 3. Quantidade de nutrientes na parte aérea do arroz colhido na maturação dos grãos

\begin{tabular}{|c|c|c|c|c|c|c|c|c|c|c|c|}
\hline \multirow{2}{*}{ Tratamento } & \multicolumn{11}{|c|}{ Nutrientes } \\
\hline & $\mathbf{N}$ & $\mathbf{P}$ & $\mathbf{K}$ & $\mathbf{C a}$ & $\mathbf{M g}$ & $\mathbf{S}$ & Mn & Zn & $\mathbf{F e}$ & B & $\mathrm{Cu}$ \\
\hline & \multicolumn{9}{|c|}{ - mg/duas plantas } & \multicolumn{2}{|c|}{ - $\mu \mathrm{g} /$ duas plantas - } \\
\hline Completo & $1715 a$ & $385,6 a$ & $989 a$ & 793abcd & $520,6 a$ & $435,5 \mathrm{~cd}$ & $25,6 a$ & $16,4 a$ & $67,7 b c$ & 1830ab & $1104 a$ \\
\hline Testemunha & $1082 \mathrm{bc}$ & $236,5 b$ & $372 c$ & $449 \mathrm{e}$ & $303,2 d$ & $385,8 \mathrm{~cd}$ & $18,6 a b$ & $8,4 c$ & $58,8 b c$ & $1463 a b$ & $501 c$ \\
\hline$-\mathrm{N}$ & $1019 \mathrm{C}$ & $325,8 \mathrm{ab}$ & $651 b c$ & 551de & $316,0 \mathrm{~cd}$ & $362,6 d$ & $16,8 \mathrm{~b}$ & $9,3 b c$ & $50,3 c$ & $1233 \mathrm{~b}$ & $655 b c$ \\
\hline$-P$ & $1619 a b$ & $289,4 a b$ & $1064 a$ & 85labc & 497,5ab & $505,9 \mathrm{~cd}$ & $22,8 a b$ & $13,5 \mathrm{ab}$ & $69,8 b c$ & $2370 a$ & $1008 a$ \\
\hline$-K$ & $1537 a b c$ & $354,8 \mathrm{a}$ & $371 c$ & 602 cde & $528,1 a$ & $534,0 \mathrm{~cd}$ & $18,8 \mathrm{ab}$ & $12,2 \mathrm{abc}$ & $60,3 b c$ & 1461ab & $818 a b$ \\
\hline$-S$ & $1674 a$ & $413,7 a$ & $973 a$ & $772 \mathrm{bcd}$ & $504,8 \mathrm{ab}$ & $495,8 \mathrm{~cd}$ & $22,3 a b$ & $13,5 \mathrm{ab}$ & $83,5 b$ & $1580 a b$ & $938 a b$ \\
\hline -Calcário & $1553 \mathrm{abc}$ & $328,0 a b$ & $913 a b$ & 681 cde & $361,5 \mathrm{bcd}$ & $568, \mathrm{obc}$ & $23,6 a b$ & $13,1 a b c$ & $116,5 \mathrm{a}$ & $1817 a b$ & $859 a b$ \\
\hline$-B$ & 1522abc & $383,9 a$ & $1059 a$ & $840 a b c$ & 552,8 & $529,2 \mathrm{~cd}$ & $26,2 a$ & $11,1 b c$ & $64,7 \mathrm{bc}$ & $1033 \mathrm{~b}$ & $915 a b$ \\
\hline$-\mathrm{Cu}$ & $1858 a$ & $350,7 a b$ & $884 a b$ & 939ab & $460,4 a b c$ & $761,9 a$ & $19,5 a b$ & $8,4 c$ & $50,33 c$ & 1761ab & $967 a$ \\
\hline$-Z n$ & $1914 a$ & $361,7 a$ & $817 a b$ & $832 a b c$ & 434,1abcc & $796,9 a$ & $20,2 a b$ & $9,4 b c$ & $66,7 \mathrm{bc}$ & 1348ab & 1046a \\
\hline -Mo & 1731a & $354,3 a$ & 818ab & $813 a b c$ & 492,7ab & $769,0 a$ & 19,7ab & $10,3 b c$ & $66,3 b c$ & 1462ab & $1040 a$ \\
\hline -Co & $1756 a$ & $342,2 a b$ & 934ab & $1030 a$ & $502,8 a b$ & $740,8 a b$ & $20,4 a b$ & $10,3 b c$ & $64,2 b c$ & $1715 a b$ & $989 a$ \\
\hline C.V. (\%) & 14,92 & 7,48 & 13,87 & 13,15 & 12,95 & 13,57 & 16,49 & 16,75 & 18,18 & 27,89 & 13,67 \\
\hline
\end{tabular}

Valores seguidos pelas mesmas letras, em cada coluna, não diferem, significativamente, entre si ao nível de 5\% pelo teste de Tukey.

produção de matéria seca pela parte aérea do arroz naquele tratamento.

Na omissão do P, não houve diferenças significativas no acúmulo de nutrientes em relação ao completo, inclusive de zinco. Vários trabal hos na literatura, como os desenvolvidos por Lopez G. \& Malavolta (1974) e Fageria \& Zimmerman (1979), mostraram que a deficiência de Zn pode estar associada a altos níveis de P disponíveis no solo, podendo também ser induzida pela aplicação de adubos fosfatados.

No tratamento em que se omitiu o K, seu acúmulo foi significativamente menor do que o completo. As relaçōes $\mathrm{K} / \mathrm{Ca}$ e $\mathrm{K} / \mathrm{Mg}$ foram 2,0 e 2,7 vezes menores, respectivamente, quando comparadas às do tratamento completo.

Deve-se ainda ressaltar a importância do calcário como fonte, principalmente, de Mg nesse solo, já que, com sua omissão, o acúmulo de Mg pela parte aérea do arroz foi significativamente, inferior ao do tratamento completo.

Nos tratamentos em que se omitiram os micronutrientes só foram observados resultados sensíveis com o Zn, cuja ausência concorreu para diminuir, significativamente, o acúmulo em rel ação ao tratamento completo.

\section{CONCLUSÕES}

1. A omissão de $\mathrm{N}$ e $\mathrm{K}$ reduziu a produção de matéria seca da parte aérea do arroz, indicando a necessidade do uso de fertilizantes contendo esses nutrientes no sol o orgânico de Macaé (RJ ).

2. Não houve decréscimo na produção de matéria seca pela ausência de P, S, B, Zn, Cu, Moe Co, indicando que suas disponibilidades no solo utilizado foram adequadas.
3. Não houve redução na produção de matéria seca pela omissão da calagem.

4. Na ausência de $N, K$ e Zn, seu acúmulo na parte aérea das plantas de arroz foi significativamente inferior ao do tratamento completo.

\section{LITE RATURA CITADA}

ABREU, C.A. de \& LOPES, A.S. Identificação de deficiências de macronutrientes em três solos de várzeas de Minas Gerais. Pesq. agropec. bras., Brasília, 22:1215-1224, 1987.

ANDRADE , A.X. A produção do arroz e a influência dos fatores climáticos. Lav. Arroz, Porto Alegre, 44:(397)3-4, 1991.

BASTOS, F.J . de M. Efeito do ferro, gesso agrícola e calcário na produção de arroz (Oryza sativa L.) em solos de várzea inundados. Lavras, Universidade Federal de Lavras, 1993. 90p. (Dissertação de Mestrado)

BLANCHAR, R.W.; REHM, G. \& CALDWELL, A.C. Sulfur in plant material digestion with nitric and perchloric acids. $\mathrm{S}$. Sci. Soc. Am. Proc., Madison, 29:71-72, 1965.

BLANCO, H.G.; WUTKE, A.C.P.; AMARAL, A.Z. do; IGUE, K. \& VERDADE, F.C. Fertilidade de alguns solos de várzea do Vale do Paraíba. Bragantia, Campinas, 23:55-62, 1964.

COMISSÃO DE FERTILIDADE DO SOLO DO ESTADO DE MINAS GERAIS. Recomendações para o uso de corretivos e fertilizantes em Minas Gerais. 4a aproximação. Lavras, Nagy Ltda. 1989. 159p.

EL-HUSNY, J.C. Limitações nutricionais para a cultura do feijoeiro (Phaseol us vulgaris L.) em um solo do Norte de Minas Gerais. Lavras, Universidade Federal de Lavras, 1992. 151p. (Dissertação de Mestrado)

EMPRESA BRASILEIRA DE PESQUISA AGROPECUÁRIA. Serviço Nacional de Levantamento e Conservação de Solos. Manual de métodos de análise de solo. Rio de J aneiro, EMBRAPA-SNLCS, 1979. 
FAGERIA, N.K. \& ZI MMERMANN, F.J .P. I nteração entre fósforo, zinco e calcário em arroz-de-sequeiro. R. bras. Ci. Solo, Campinas, 3:88-92, 1979.

GUILHERME, L.R.G. Calagem e inundação em solos de várzeas cultivadas com arroz: alterações em pH, nitrogênio, fósforo e enxofre. Lavras, Universidade Federal de Lavras, 1990. 112p. (Dissertação de Mestrado)

J ACKSON, M.L. Análise química de suelos. 2aed. Barcelona, Ed. Omega, 1970. 662p.

LEITE, N.; GARGANTINI, H. \& HUNGRIA, L.S. Efeitos das adubações nitrogenadas e fosfatada em cultura de arroz, em condições de várzea irrigada. Bragantia, Campinas, 29:115-125, 1970a.

LEITE, N.; GARGANTINI, H.; HUNGRIA, L.S. \& I GUE, T. Efeitos do nitrogênio, fósforo, calcário e micronutrientes em cultura de arroz irrigada no Vale do Paraíba. Bragantia, Campinas, 29:273-285, 1970b.

LOPEZ G., O.E.\& MALAVOLTA, E. Estudos sobre as relações entre zinco e fósforo na nutrição da planta. Anais da ESALQ, Piracicaba, 31:467-483, 1974.

MALAVOLTA, E. \& MURAOKA, T. Avaliação do estado nutricional e da fertilidade do solo; métodos de vegetação - diagnose por subtração, em vasos. Piracicaba, CENA - USP, 1985. 7p.
MESQUITA, H.A. de. Efeito do gesso e do cal cário em solo aluvial cultivado com arroz (Oryza sativa L.). Lavras, Universidade Federal de Lavras, 1993. 81p. (Dissertação de Mestrado)

NOVAIS, R.F. de.; BRAGA, J .M.; DEFELIPO, B.V.; FAJ ARDO, C.M. \& AMARAL, F.A.L. Níveis ótimos de NPK na adubação de arroz irrigado em solos de várzea da Zona da Mata, Minas Gerais. Rev. Ceres, Viçosa, 19:63-77, 1972.

PAULA, M.B. de.; CARVALHO, J.G. de.; SOARES, A.A. \& NOGUEIRA, F.D. Avaliação da fertilidade de um solo de várzea (glei húmico) para a cultura do arroz. Pesq. agropec. bras., Brasília, 25:571-577, 1990.

PEREIRA, L.F. Efeitos da aplicação de cal cário, fósforo e zinco no crescimento e nutrição do trigo (Triticum aestivum L.) e arroz (Oryza sativa L.) em dois sol os de várzeas do Estado de Minas Gerais. Lavras, Universidade Federal de Lavras, 1985. 135p. (Dissertação de Mestrado)

PIMENTEL GOMES, F. A estatística moderna na pesquisa agropecuária. Piracicaba, POTAFOS, 1984. 160p.

RAIJ , B.V.; QUAGGIO, J.A.; CANTARELA, H.; FERREIRA, M.E.; LOPES, A.S. \& BATAGLIA, O.C. Análise química do solo para fins de fertilidade. Campinas, Cargill, 1987. 170p.

SANCHEZ, P.A. Properties and management of soils in thetropics. New York, J. Wiley, 1976. 618p. 